\title{
Digital image processing methods for estimating leaf area of cucumber plants
}

\author{
Uoc Quang Ngo ${ }^{1,2}$, Duong Tri Ngo ${ }^{1}$, Hoc Thai Nguyen ${ }^{1}$, Thanh Dang Bui ${ }^{2}$ \\ ${ }^{1}$ Faculty of Engineering, Vietnam National University of Agriculture, Hanoi, Vietnam \\ ${ }^{2}$ Institute for Control Engineering and Automation, Hanoi University of Science and Technology, Hanoi, Vietnam
}

\begin{abstract}
Article Info
ABSTRACT

Article history:

Received Aug 3, 2021

Revised Oct 29, 2021

Accepted Nov 27, 2021

Increasingly emerging technologies in agriculture such as computer vision, artificial intelligence technology, not only make it possible to increase production. To minimize the negative impact on climate and the environment but also to conserve resources. A key task of these technologies is to monitor the growth of plants online with a high accuracy rate and in non-destructive manners. It is known that leaf area (LA) is one of the most important growth indexes in plant growth monitoring system. Unfortunately, to estimate the LA in natural outdoor scenes (the presence of occlusion or overlap area) with a high accuracy rate is not easy and it still remains a big challenge in eco-physiological studies. In this paper, two accurate and nondestructive approaches for estimating the LA were proposed with top-view and side-view images, respectively. The proposed approaches successfully extract the skeleton of cucumber plants in red, green, and blue (RGB) images and estimate the LA of cucumber plants with high precision. The results were validated by comparing with manual measurements. The experimental results of our proposed algorithms achieve $97.64 \%$ accuracy in leaf segmentation, and the relative error in LA estimation varies from $3.76 \%$ to $13.00 \%$, which could meet the requirements of plant growth monitoring systems.
\end{abstract}

This is an open access article under the $\underline{C C B Y-S A}$ license.

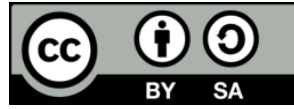

\section{Corresponding Author:}

Uoc Quang Ngo

Faculty of Engineering, Vietnam National University of Agriculture

Ngo Xuan Quang Road, Trau Quy Township, Hanoi, Vietnam

Email: nquoc@vnua.edu.vn

\section{INTRODUCTION}

Modern agriculture with high technology has been played a crucial role in feeding the growing population [1]. By efficiently utilizing the limited resources, using modern technologies in farming, farmers in modern agriculture enhance both quantity and quality of agricultural products [2]-[4]. Plant growth monitoring based on machine vision is one of such modern technologies [5]. This monitoring approach using digital cameras and suitable sensors for image acquisition. The growth analysis of plant phenotypes in a nondestructive manner is conducted automatically [6]-[8]. However, some plant features, such as stem diameter, leaf number, and leaf area are not measured easily by such non-contact measurement methods [9]. Therefore, improving the quality of plant features estimation is one of the primary concerns of present-day plant management [10]-[15].

Leaf is one of the most important photosynthetic organs of a plant [16]. Hence, leaf area is an important factor in measuring the growth and productivity of plants. Consequently, the precise determination of leaf area is basic in many plant physiological studies [17]-[20]. In fact, the leaf area is traditionally measured by regression equation methods [21], grid counting methods [22], gravimetric method [23], and 
some leaf area meters (planimeter) [24], [25]. However, most of these methods are destructive (they require the excision of leaves, which may damage the growth of plants), and provide off-line performance analysis. Though these traditional methods have high accuracy, they require expensive equipments and timeconsuming experiments. Fortunately, some nondestructive methods for LA estimation [26]-[29] have been being developed rapidly to minimize the disadvantages of these destructive methods. It is proven that the nondestructive methods achieve better performances, such as faster, cheaper, and provide real-time experimental analysis than the destructive methods.

Nowadays, nondestructive measurement methods based on digital image analysis are widely used in many growth models. The authors in [27] have focused on measuring cucumber parameters based on machine vision technology. It is a real non-destructive online measurement method that used digital cameras to capture the canopies of cucumber plants. The image parameters of cucumber plants are combined with manual measurements to construct some inversion models for cucumber growth parameters. The results show that with high coefficients of determination between manual measurements and inversion values of cucumber parameters, these inversion models are able to be a non-destructive online method for measuring cucumber parameters.

Wang et al. [26] developed a new image segmentation method based on the Chan-Vese (C-V) model and the Sobel operator. In order to improve the segmentation precision of overlapping cucumber leaves, the procedure of the image segmentation method is described as follows: First, the background is mitigated based on a threshold with respect to the relative levels of green in the image. Using the $\mathrm{C}-\mathrm{V}$ model, the contour of the target leaf in the upper layer is extracted. Then, the leaf edges of the target leaf are detected by a gradient operator with eight directions. Finally, the combination of the results obtained by the $\mathrm{C}-\mathrm{V}$ model and the Sobel operator is used to extract the overlapping target leaf. The experimental results show that with a low level of the mean error rate $(0.0428)$, the proposed method is successful in extracting the target leaf from complicated background images of cucumber plants.

Ibrahim et al. [30], two image segmentation methods such as k-mean clustering and region growing have been used for automatic flood detection. The simulation results reveal that the region growing using gray images achieved higher segmentation accuracy than that of k-mean clustering method. In [31], a system for detection and classification of various pest attacks and infection on plants is introduced. For segmentation of preprocessed sample, an image segmentation method based on hybrid watershed segmentation with extended K-Means clustering is proposed. The experimental results show that the proposed approach achieved higher accuracy rates than some existing approaches in disease/pest detection. More recently, Liu et al. [32] proposed an instance segmentation method, which used an improved version of mask region-based convolutional neural network (Mask RCNN) to detect cucumber fruits in pixel level. The experimental results reveal that the proposed improved Mask RCNN achieves high accuracy in detecting cucumber fruits. The precision and recall rates can reach $90.68 \%$ and $88.29 \%$, respectively. All studies mentioned above show that LA of cucumber plants can be determined by such nondestructive methods with high accuracy. This important LA information helps growers having proper time irrigation, cultivation, therefore, get better yields. In this paper, we have focused on improving the accuracy of cucumber leaf area estimation, which could provide a valuable resource for managing the cucumber plant.

\section{RESEARCH METHOD}

According to Figure 1, the flowing chart of cucumber leaf area estimation is used to improve the research and experiment comparison. The flowchart illustrates the various steps of a process that a captured image is used for LA estimation. There are two different estimation techniques for top-view and side-view images. Each step of the process is described in more detail in following subsections.

\subsection{Background elimination}

To improve the segmentation accuracy of overlapping target leaves, some approaches [26], [33] have focused on removing background (the non-leaf areas such as soil, stems, and petals). On considering the difference of colour between background areas and foreground areas (leaf areas) [33], the authors in [26] used the levels of the green channel in the RGB colour space to remove all pixels in non-leaf areas. In that study, the relationship of pixel colour channels for a green leaf is determined in (1).

$$
\left(G_{\text {value }}-B_{\text {value }}>\theta_{1}\right) \cap\left(G_{\text {value }}-R_{\text {value }}>\theta_{2}\right)
$$

where $R_{\text {value }}, B_{\text {value }}$, and $G_{\text {value }}$ are value of red, blue, and green component, respectively. $\theta_{1}, \theta_{2}$ are the $R_{\text {value }}$ and $B_{\text {value }}$ control parameters, respectively. These parameters vary in different species of plant, e.g., they are chosen $\theta_{1}=10$, and $\theta_{2}=15$ for leaf segmentation of cucumber leaves. In this way, we could 
remove all non-leaf areas by just keeping pixels whose relationship of colour channel is green. Unfortunately, if the difference between the background colour and the foreground colour is not apparent or the colours of leaves change in the autumn, this approach might not work properly.

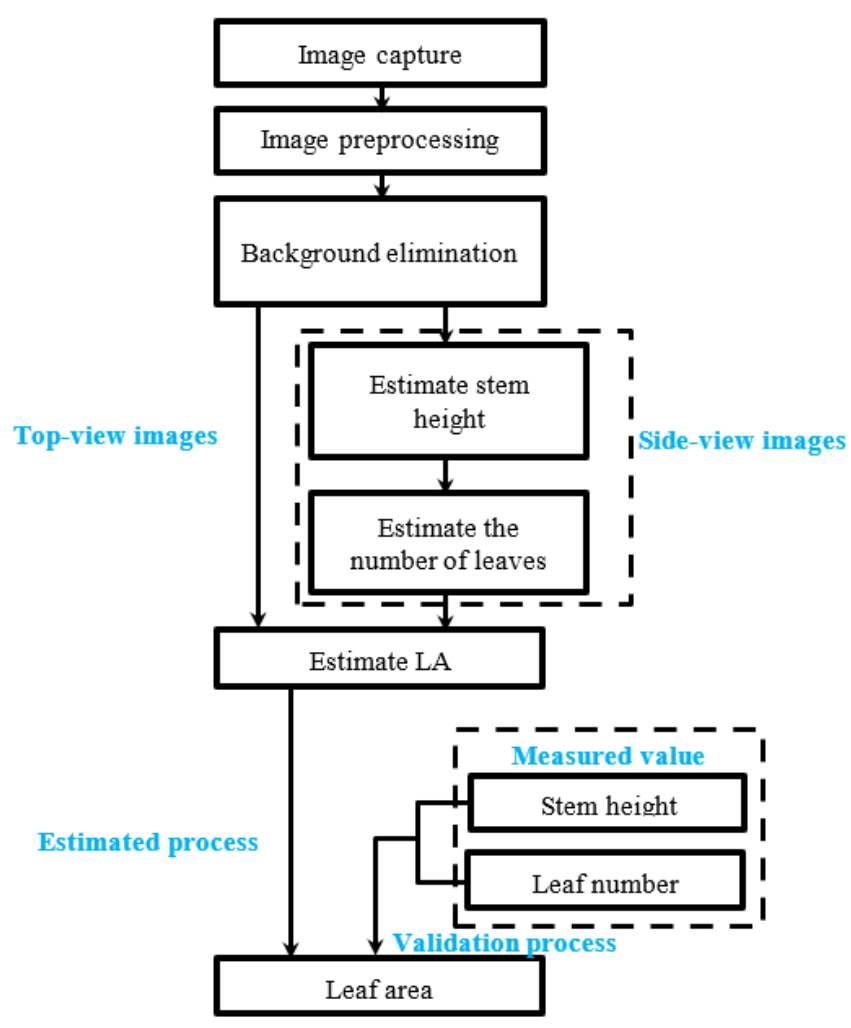

Figure 1. The flowing chart of cucumber leaf area estimation

Fortunately, the Mahalanobis distance [34] is an efficient alternative approach for background image removal that mitigates these above-mentioned limitations. The basic idea of this image segmentation method is to compute the distance between colour intensities of pixels and their neighbouring pixels within an ellipsoidal region. One pixel becomes a member of a group if its Mahalanobis distance is smaller than a threshold. In this way, the Mahalanobis distance based method for image background removal is expected to improve the quality and reduce the false alarms [35].

In fact, many Mahalanobis distance based image background removal algorithms are proposed [36], [37]. In [35], for weed leaf image segmentation, each pixel of the image has been classified as a weed area pixel or not based on the Mahalanobis distance from the plant colour class and a colour threshold value. The experimental results show that $84 \%$ of the weed leaves were correctly segmented. It is clear that this segmentation accuracy is quite high and acceptable for weed segmentation with multiple leaves in an image. However, not many of these studies consider how to determine an optimal threshold of each sub-region for leaf segmentation. It might increase the accuracy of image segmentation [38]. To do this, in this paper, we propose a Modified Mahalanobis Distance based method (MMD) for background image removal.

Without loss of generality, let denote the color value of a pixel in a color image. We assume that the color of each pixel is the normal distribution.

In order to build the foreground model, some initial frames that have only foreground (the leaf image of considered plants) are analyzed. Let denote the number of such initial frames. The mean of the colour at every pixel is determined in the (2) [39].

$$
\mu=\frac{1}{k} \sum_{k} x_{k}
$$

And the covariance is computed as follows:

$$
C=\frac{1}{k} \sum_{k}\left(x_{k}-\mu_{k}\right)\left(x_{k}-\mu_{k}\right)^{T}
$$


after making the background model, the Mahalanobis distances of every pixel are calculated using (4):

$$
d=\sqrt{(x-\mu)^{T} C^{-1}(x-\mu)}
$$

Check if $d>\theta_{0}$, where $\theta_{0}$ is predefined threshold, that pixel is classified as foreground, otherwise, it belongs to background. The problem here is how to determine the optimal threshold $\left(\theta_{0}^{*}\right)$ for leaf segmentation of different plant?. Xia et al. [37] $\theta_{0}^{*}=10,40,7$ respectively for pepper, old paprika, and young paprika after testing leaf images of these plants. Unfortunately, these leaf segmentation approaches based on a global threshold of the entire image may lead to low accuracy and a high rate of false-positive alarms. Therefore, in order to improve the accuracy and effectiveness in leaf segmentation, the threshold should change adaptively depending on properties of each sub-image. To do this, the authors in [40] implemented a new segmentation algorithm with the following steps. First, the standard deviation of the grey level of the whole image, which is named global standard deviation, is computed. Then the image is divided into four equal regions. Each sub-region is continuously split into four sub-regions if its standard deviation of the grey level (namely local standard deviation) is higher than the global standard deviation. This procedure is recursively continued until the local standard deviation is less than or equal to the global standard deviation of image grey level. The threshold of each sub-region is determined based on the local properties of the image by using IsoData algorithm [41].

In this study, we combine the Mahalanobis distance algorithm and optimizing threshold algorithm in [40] to propose a modified Mahalanobis distance-based method for background image removal. The details of our proposed MMD algorithm are described in algorithm 1.

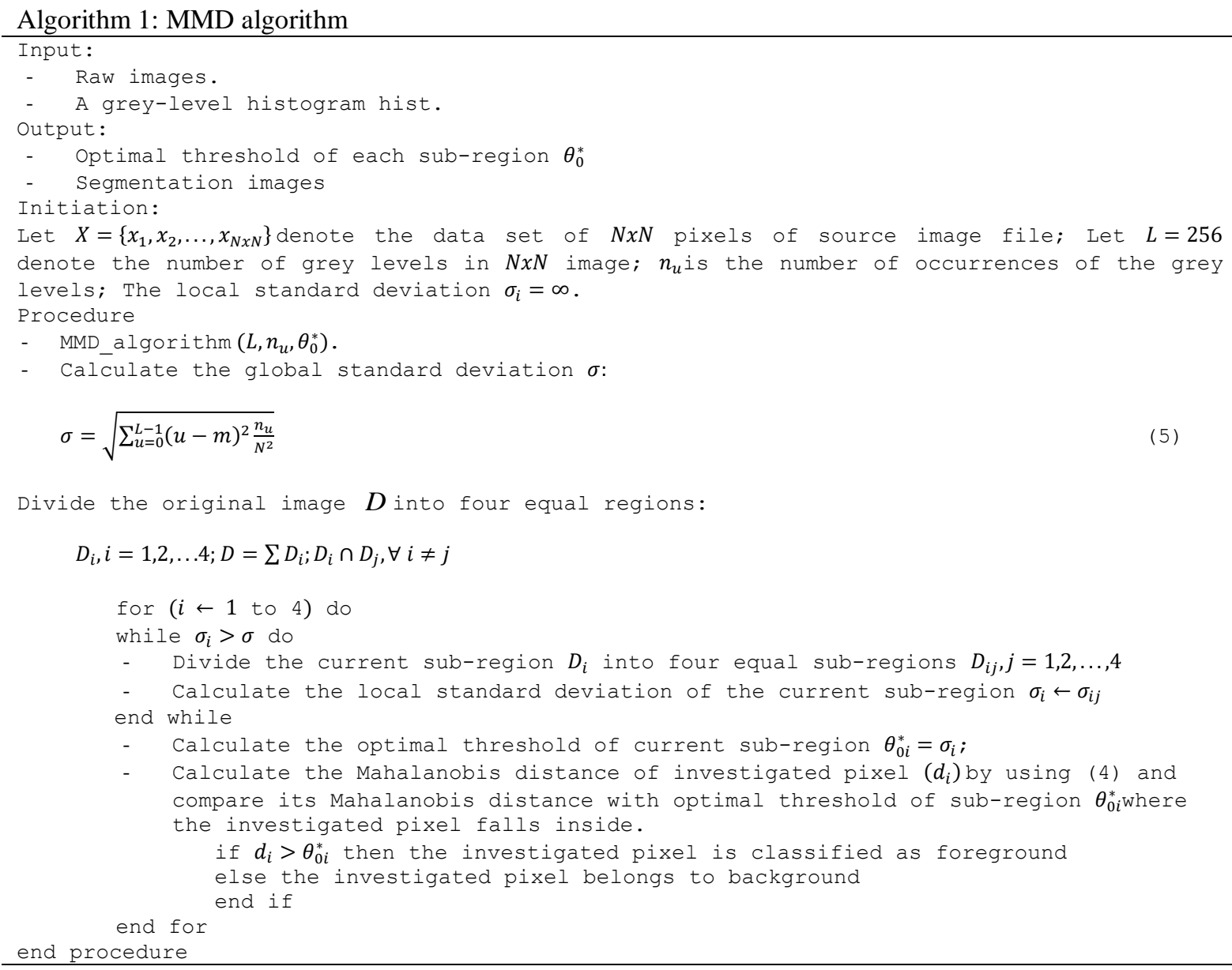

For evaluating the quality of our proposed image segmentation algorithm, manual segmentation images were used to compare with the results of this segmentation algorithm. The following performance indices have been used:

a) There are four possible outcomes when classifying a pixel: true positive, false positive, true negative and false negative [42]. They are described more in detail in Figure 2. 
- $\quad$ True positive (TP): Pixels of leaf areas are classified correctly as leaf pixels.

- $\quad$ False positive $(F P)$ : Pixels in non-leaf areas are misclassified as leaf pixels.

- $\quad$ True negative $(T N)$ : Pixels in non-leaf areas are classified correctly as non-leaf pixels.

- $\quad$ False negative $(F N)$ : Pixels of leaf areas are misclassified as non-leaf pixels.

b) The false negative rate (FNR) is computed as given in (6).

$$
F N R=\frac{F N}{T P+F N}
$$

c) The false positive rate (FPR) is computed as given in (7).

$$
F P R=\frac{F P}{F P+T N}
$$

d) The error rate (ER) is mathematically defined in (8).

$$
E R=\frac{F N+F P}{T P+T N+F P+F N}
$$

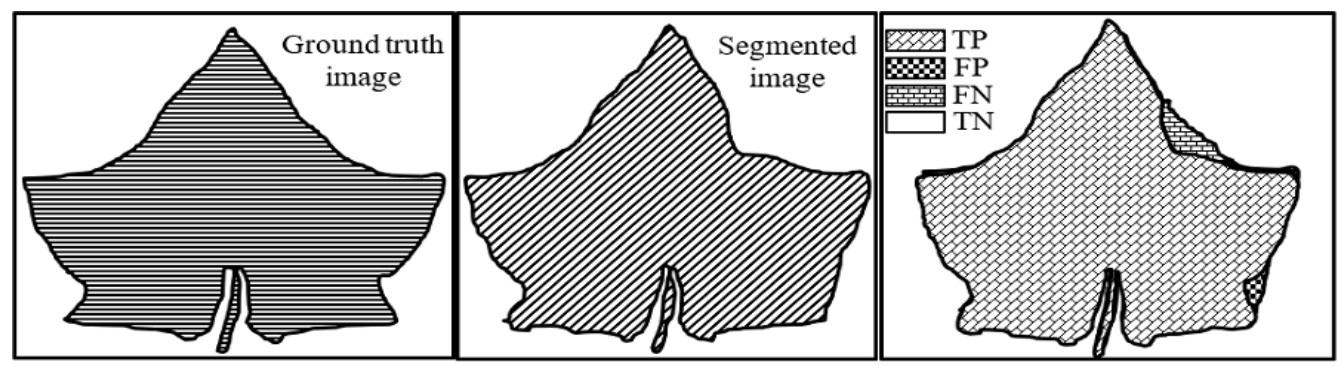

Figure 2. Schematic illustrating the definition of four possible outcomes when classifying a pixel

\subsection{Measurement of leaf area}

Nondestructive leaf area estimation with a high accuracy rate and in a small running time is critical in many specific fields of plant growth modelling (e.g., plant physiological and ecological experiments [43]). Leaf area may be used for leaf area index calculation [9], [44] which is likely to model vegetation productivity and photosynthetic capacity [45], [46]. Moreover, LA is a very useful parameter in plant growth and morphological studies of the plants [47].

In this study, after removing the background, the leaf areas are measured by counting leaf pixels and comparing them with red calibration pixels. In this way, our proposed approach is able to estimate the leaf areas automatically while mitigating the need for manual operations for camera distance calculations. However, there are some overlap areas of the leaves, which may be considered as one layer of leaves. Additionally, the difference between green leaves and green objects may not be distinguished correctly if we only use top view images of the plant. Therefore, in our proposed algorithm, we use both top view and side view images of the plants to estimate the leaf area.

The following steps are used to estimate the leaf area of the cucumber plant in the top-view image.

Step 1: Convert the original image into a binary image.

Step 2: Count the number of pixels in the calibration area $\left(P_{c l}\right)$ (The size of the calibration area given in this paper is $25 \mathrm{~cm}^{2}$ ).

Step 3: Count the number of pixels in the leaf image $\left(P_{l}\right)$ of the considered plant.

Step 4: The leaf area can be obtained by (9).

$$
L A_{e}=\frac{25 P_{l}}{P_{c l}}
$$

The side-view images of plants are used to estimate the main stem length of plants and then to estimate the number of leaves. According to the number of leaves, we estimate the leaf area of the plant. The following steps are used to estimate the leaf area of cucumber plant in the side-view image:

Step 1: Convert the original image into a binary image.

Step 2: Skeleton extraction of cucumber plant by using Zhang-Suen algorithm [48]. The main stem of cucumber is described in white pixels. 
Step 3: Calculate the length of the main stem of cucumber plant: Scan the image from the bottom-left corner to the top-right corner of the image frame to catch and locate all white pixels. The length of the main stem of the cucumber plant is cumulative sum of Euclidean distances between these white pixels.

Step 4: Count the number of nodes along the stem of the cucumber plant. And then estimate the number of leaves on the main stem.

Step 5: According to the results given in [49] and our manual measurements given in Table 1, the estimated leaf area $\left(L A_{e}\right)$ is the cumulative sum of leaf area from the oldest leaf (the first leaf) to the youngest leaf on the cucumber stem.

Table 1. Measured data

\begin{tabular}{|c|c|c|c|c|c|c|c|c|c|c|c|c|c|c|c|}
\hline \multirow[b]{2}{*}{ DAT } & \multirow{2}{*}{$\begin{array}{l}\text { Leaf } \\
\text { number }\end{array}$} & \multirow[b]{2}{*}{1 st } & \multirow[b]{2}{*}{ 2nd } & \multirow[b]{2}{*}{$3 \mathrm{rd}$} & \multicolumn{5}{|c|}{ Average of leaf area $\left(\mathrm{cm}^{2}\right)$} & \multirow[b]{2}{*}{ 9th } & \multirow[b]{2}{*}{ 10th } & \multirow[b]{2}{*}{ 11th } & \multirow[b]{2}{*}{12 th } & \multirow[b]{2}{*}{$L A_{m}$} & \multirow[b]{2}{*}{$\begin{array}{c}L_{m} \\
(\mathrm{~cm})\end{array}$} \\
\hline & & & & & 4 th & 5 th & 6th & 7th & 8 th & & & & & & \\
\hline 10 & 2 & 07.25 & 100.07 & & & & & & & & & & & 207.32 & 12.35 \\
\hline 12 & 2.5 & 08.44 & 100.70 & 46.25 & & & & & & & & & & 255.39 & 18.21 \\
\hline 14 & 3.62 & 10.92 & 101.20 & 92.48 & 66.51 & & & & & & & & & 371.10 & 23.89 \\
\hline 16 & 4.45 & 11.97 & 101.93 & 94.17 & 90.05 & 64.82 & & & & & & & & 462.94 & 30.21 \\
\hline 18 & 5.61 & 13.72 & 103.21 & 95.22 & 89.91 & 74.38 & 58.39 & & & & & & & 534.83 & 35.72 \\
\hline 20 & 6.45 & 15.74 & 104.91 & 96.68 & 92.35 & 75.36 & 80.38 & 48.14 & & & & & & 613.56 & 41.55 \\
\hline 22 & 7.65 & 21.26 & 106.30 & 98.10 & 92.96 & 77.38 & 97.13 & 90.99 & 78.81 & & & & & 762.92 & 47.42 \\
\hline 24 & 8.8 & 27.95 & 106.73 & 98.41 & 93.65 & 78.03 & 110.26 & 93.12 & 108.91 & 90.42 & & & & 907.50 & 53.71 \\
\hline 26 & 9.75 & 33.82 & 122.70 & 112.94 & 110.99 & 88.85 & 117.83 & 103.46 & 118.24 & 121.47 & 83.98 & & & 1114.27 & 59.29 \\
\hline 28 & 10.92 & 37.68 & 130.18 & 136.44 & 118.38 & 103.12 & 119.98 & 108.30 & 126.41 & 128.70 & 116.52 & 102.66 & & 1328.38 & 64.35 \\
\hline 30 & 11.95 & 41.61 & 135.96 & 147.32 & 131.55 & 129.33 & 123.94 & 123.98 & 135.20 & 135.86 & 127.79 & 130.02 & 115.4 & 1578.01 & 70.65 \\
\hline
\end{tabular}

\subsection{Experiments}

Image acquisition system: The cucumber plants are labelled daily with QR codes which contain species, day after transplanting of cucumber. As depicted in Figure 3, our machine vision system consists of two cameras (Nikon camera D3500 DX), a personal computer (Core i7-7700 CPU, OS: MS Windows 10) and our image processing program is developed in Python software. The top-view image acquisition sytem in Figure 3(a) is a checkerboard target with known dimension is used as calibration area. The other calbiration area is also used the side-view image acquisition sytem in Figure 3(b). As depicted in Figures 4(a) and (b), all red squares are used as referenced area for leaf area estimation.

Experimental design; The experiments are conducted from March 1 to April 30, 2021 in an EastWest, polyethylene-covered greenhouse at the Vietnam National University of Agriculture (lat.21.0079764'N, long. 105.9335549E), Vietnam. The experimental greenhouse had two plots and 200 cucumber plants (Cucumis sativus L. cv. Deltastar). The distance between two adjacent plants is $30 \mathrm{~cm}$. Four plants in one plot are selected randomly for vegetation measurements. Images were taken on sunny days in direct sunlight. The image capture time was once at 11h:30 AM in every two days. The experimental images are shown in Figure 4 with top-view image in Figure 4(a) and side-view image in Figure 4(b).

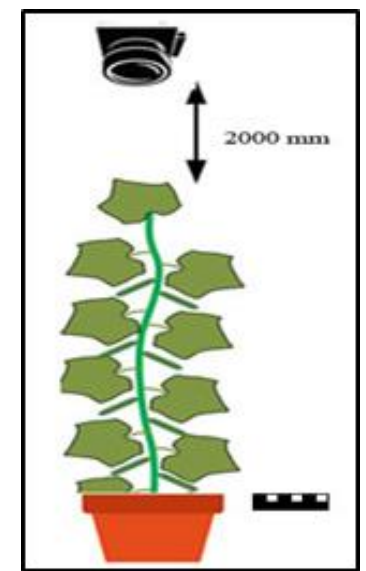

(a)

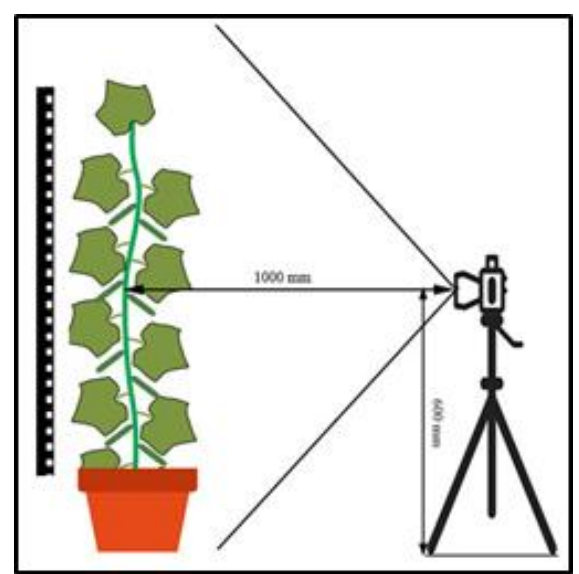

(b)

Figure 3. Block diagram of image acquisition system: (a) top-view, and (b) side-view 


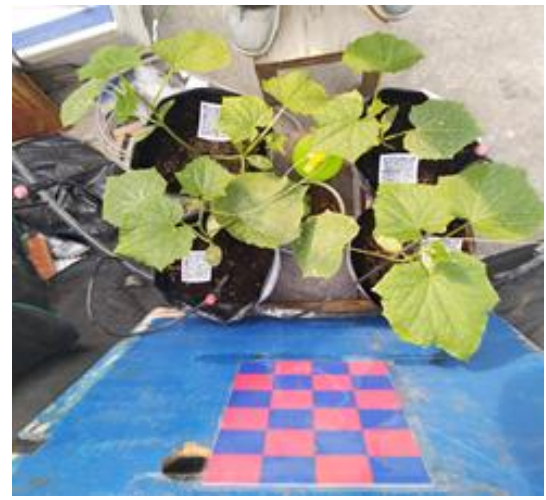

(a)

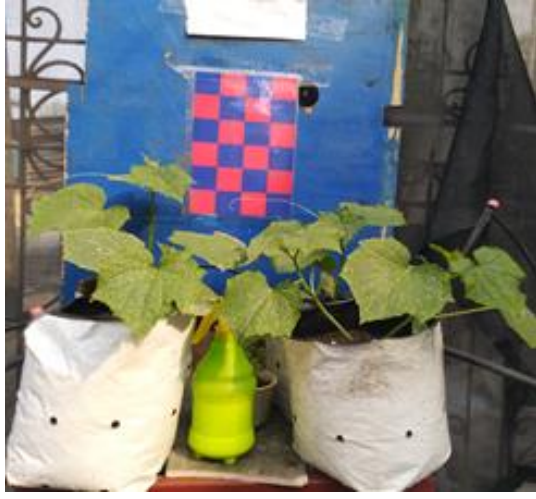

(b)

Figure 4. Samples of cucumber plant images: (a) top-view, and (b) side-view

In this paper, we used the manual measurements which serve as references for evaluating the performance of our proposed algorithms. The manual measurements included leaf number, the length of the main stem $\left(L_{m}\right)$, the width and length of each leaf. According to the results given in [49], in this paper the measured value of leaf area $\left(L A_{m}\right)$ was carried out as given in (10).

$$
\begin{aligned}
& L_{s}=-29.96+0.849 * l_{w s} * l_{l s} \\
& L A_{m}=\sum_{s} L_{s}
\end{aligned}
$$

Where $l_{w s}$ and $l_{l s}$ are measured width and length of leaf at position sth on cucumber plant, respectively. 80 cucumber plants were randomly selected for manual measurement. Leaves at different positions ( $s$ from 1st (the oldest leaf) to 12th leaf) are measured 50 times and the average values of these parameters are given in Table 1.

\section{RESULTS AND DISCUSSION}

\subsection{Segmentation results}

Eighty cucumber plants were used for leaf segmentation. In Figure 5, the segmentation results of top-view images are shown. It can be seen that the original image in Figure 5(a), is divided into some subregions. In each sub-region, an optimal threshold is chosen for comparison with the mahalobis distance of all pixels falling inside this sub-region.

In Figure 5, there are 3 separate layers with different colour ranges. The first layer is the leaf area of cucumber plants in Figure 5(b). In this layer, all pixels were considered as part of cucumber leaves. The second layer denotes the calibration area in Figure 5(c), which is used for leaf area calculation later. The ambient layer in Figure 5(d) represents other objects in the image.

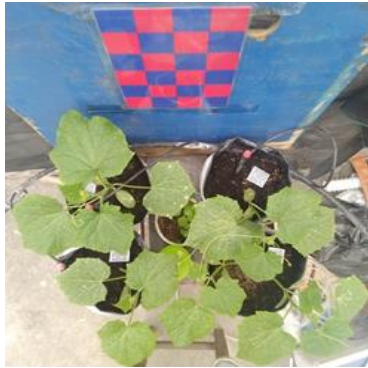

(a)

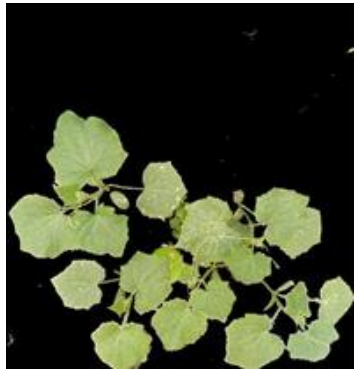

(b)

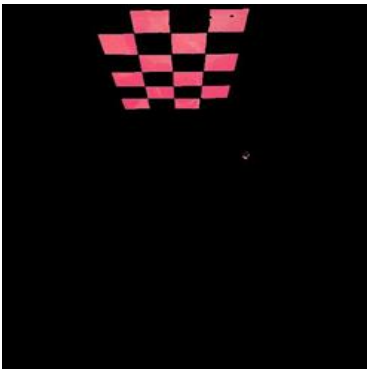

(c)

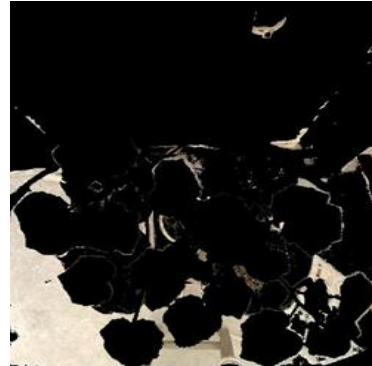

(d)

Figure 5. Background removal in top-view image: (a) original image, (b) leaf area, (c) calibration layer, and (d) ambient layer 
For evaluation, we conduct some experiments with same conditions to measure the performance of our proposed method and compare with the results of original mahalanobis distance based method [36] for leaf segmentation. The comparsion results are given in Table 2. It is clear that with the growth of cucumber plants, the number of leaves increases. Cucumber plants with greater leaf area have a larger number of overlapping leaves. Consequently, the number of misclassified pixels also increases. The results in Table 2 show that all three aspects (FNR, FPR, and ER) increase with the growth of cucumber plants. With lower noise points in the top-view images, all three performance indices have lower increase rates than that in the side-view images in all growth stages of plants (DAT). Figure 6, shows the segmentation results in side-view image. After removing the background of the original image in Figure 6(a), the leaf layer, calibration layer, and ambient layer are segmented and depicted in Figures 6(b)-(d) respectively. The leaf layer in Figure 6(b) shows that the number of FP and FN pixels are significantly higher than that in Figure 5(b). The results of our proposed MMD algorithm indicates that the averages of FNR, FPR, ER are 0.0284, 0.0201 and 0.0236, respectively. The average of misclassified pixels in every image is $2.36 \%$. It reveals that our proposed MMD algorithm performs well and can be effectively used in most of image-processing applications.

Table 2. The comparsion results of leaf segmentation methods

\begin{tabular}{|c|c|c|c|c|c|c|c|}
\hline \multirow{2}{*}{ DAT } & \multirow{2}{*}{ Image view } & \multicolumn{3}{|c|}{ Original Mahalanobis distance-based method } & \multicolumn{3}{|c|}{ Our proposed MMD method } \\
\hline & & FNR & FPR & ER & FNR & FPR & ER \\
\hline \multirow{2}{*}{10} & Top-view & 0.2221 & 0.0364 & 0.1071 & 0.0114 & 0.0245 & 0.0195 \\
\hline & Side-view & 0.1402 & 0.0314 & 0.0748 & 0.0119 & 0.0155 & 0.0141 \\
\hline \multirow{2}{*}{12} & Top-view & 0.1890 & 0.0191 & 0.0994 & 0.0137 & 0.0144 & 0.0141 \\
\hline & Side-view & 0.1291 & 0.0240 & 0.0698 & 0.0145 & 0.0161 & 0.0155 \\
\hline \multirow{2}{*}{14} & Top-view & 0.1284 & 0.0296 & 0.0730 & 0.0153 & 0.0150 & 0.0151 \\
\hline & Side-view & 0.1775 & 0.0198 & 0.0858 & 0.0161 & 0.0161 & 0.0161 \\
\hline \multirow{2}{*}{16} & Top-view & 0.1628 & 0.0216 & 0.0774 & 0.0169 & 0.0167 & 0.0168 \\
\hline & Side-view & 0.1481 & 0.0210 & 0.0773 & 0.0177 & 0.0173 & 0.0175 \\
\hline \multirow{2}{*}{18} & Top-view & 0.2577 & 0.0251 & 0.1303 & 0.0185 & 0.0179 & 0.0181 \\
\hline & Side-view & 0.1991 & 0.0273 & 0.0958 & 0.0194 & 0.0185 & 0.0188 \\
\hline \multirow{2}{*}{20} & Top-view & 0.1550 & 0.0308 & 0.0815 & 0.0202 & 0.0190 & 0.0195 \\
\hline & Side-view & 0.1406 & 0.0240 & 0.0742 & 0.0275 & 0.0197 & 0.0228 \\
\hline \multirow{2}{*}{22} & Top-view & 0.2986 & 0.0402 & 0.1600 & 0.0259 & 0.0203 & 0.0225 \\
\hline & Side-view & 0.1779 & 0.0354 & 0.0948 & 0.0266 & 0.0208 & 0.0232 \\
\hline \multirow{2}{*}{24} & Top-view & 0.1049 & 0.0323 & 0.0617 & 0.0274 & 0.0214 & 0.0239 \\
\hline & Side-view & 0.2446 & 0.0275 & 0.1264 & 0.0290 & 0.0220 & 0.0249 \\
\hline \multirow{2}{*}{26} & Top-view & 0.3209 & 0.0437 & 0.1793 & 0.0378 & 0.0228 & 0.0289 \\
\hline & Side-view & 0.2348 & 0.0376 & 0.1293 & 0.0393 & 0.0234 & 0.0299 \\
\hline \multirow{2}{*}{28} & Top-view & 0.2788 & 0.0310 & 0.1552 & 0.0455 & 0.0241 & 0.0329 \\
\hline & Side-view & 0.1786 & 0.0288 & 0.0986 & 0.0517 & 0.0248 & 0.0360 \\
\hline \multirow{2}{*}{30} & Top-view & 0.2751 & 0.0413 & 0.1521 & 0.0637 & 0.0256 & 0.0417 \\
\hline & Side-view & 0.2145 & 0.0319 & 0.1147 & 0.0754 & 0.0264 & 0.0474 \\
\hline \multicolumn{2}{|c|}{ Average } & 0.1990 & 0.0300 & 0.1054 & 0.0284 & 0.0201 & 0.0236 \\
\hline
\end{tabular}

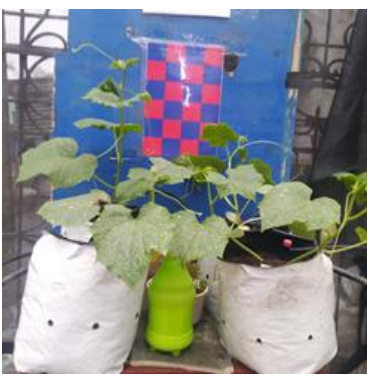

(a)

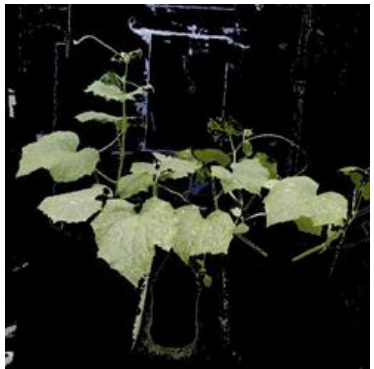

(b)

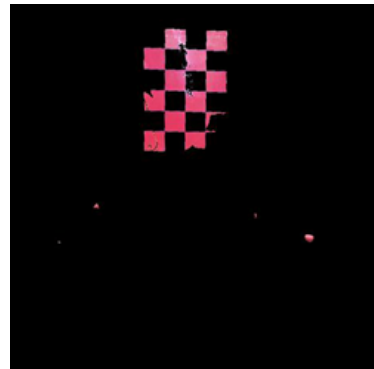

(c)

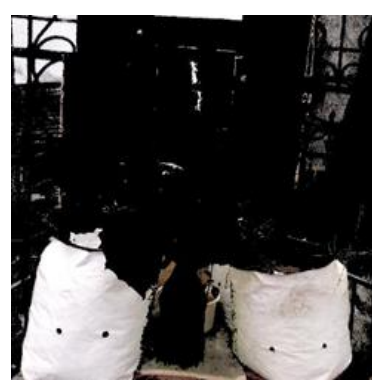

(d)

Figure 6. Background removal in side-view image: (a) original image, (b) leaf layer, (c) calibration layer, and ambient layer 
According the experimental results given in Table 2, the proposed MMD algorithm achieves higher accuracy rate, which is $8.18 \%$ greater than that of original Mahalanobis distance based algorithm. One main reason of this difference is how to chose a segmentation threshold for each window of segmentation image. In general, the original Mahalanobis distance based methods choose a segmentation threshold for all segentation images, which tends to increase the segmentation error rate in homogeneous colour regions. While in our proposed MMD algorithm, each image being segmented is splitted into smaller regions and then the segmentation threshold is determined based on image local properties. As consequence, our proposed MMD algorithm worked well even in homogeneous colour regions.

\subsection{Leaf area estimation results}

As described above, there are two approaches for LA estimation of cucumber plants. With the topview images of cucumber plants, the LA in each image is computed as given in (9). However, with side-view images we first estimated the number of leaves in each image and then estimated the LA based on manual measurements given in Table 1. In order to estimate the number of leaves in side-view images, we first find the skeleton of each cucumber plant and then count the nodes along these plants stems. Each node has a leaf. The procedure of skeleton extraction is described in Figure 7. The original image in Figure 7(a) is used for segmentation. Figure 7(b) shows the segmentation result of our proposed MMD algorithm. The background of this segmentation image is changed to white colour and given in Figure 7(c). The binary image is depicted in Figure 7(d). By using Zhang-Suen algorithm [48], the main stems of cucumber plants are given in Figure 7(e).

As described in Figure 8, there is no significant difference between the estimated and measured length of the main cucumber stem. The relative error of these two parameters varies from $4.77 \%$ to $8.47 \%$, which indicates that our proposed algorithm can achieve high accuracy in measuring the length of the main cucumber stem. The highest value of relative error is only $8.47 \%$ when DAT is 16 days.

Table 3 provides a summary of LA estimation results. It indicates that the relative error of our proposed approach increases from $3.76 \%$ to $13.00 \%$. Having larger overlapped leaf regions in top-view images, the relative errors of leaf area estimation with the same cucumber plant are normally higher than that in side-view images.

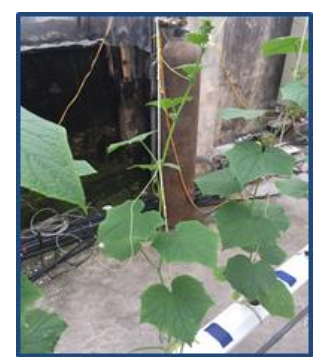

(a)

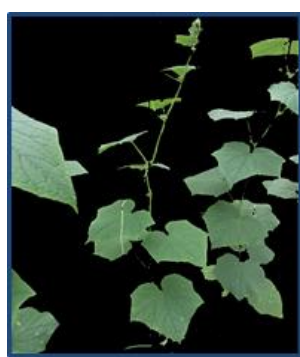

(b)

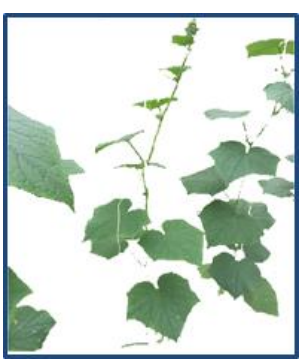

(c)

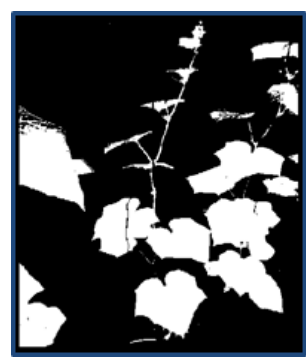

(d)

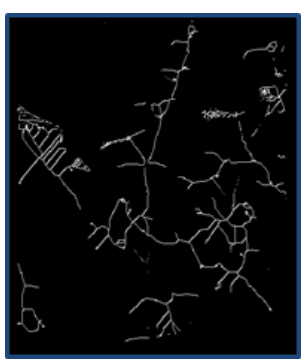

(e)

Figure 7. Schematic illustrating the process of skeleton extraction: (a) original image, (b) segmentation image, (c) white background segmentation image, (d) binary image, and (e) skeletion of cucumber plant

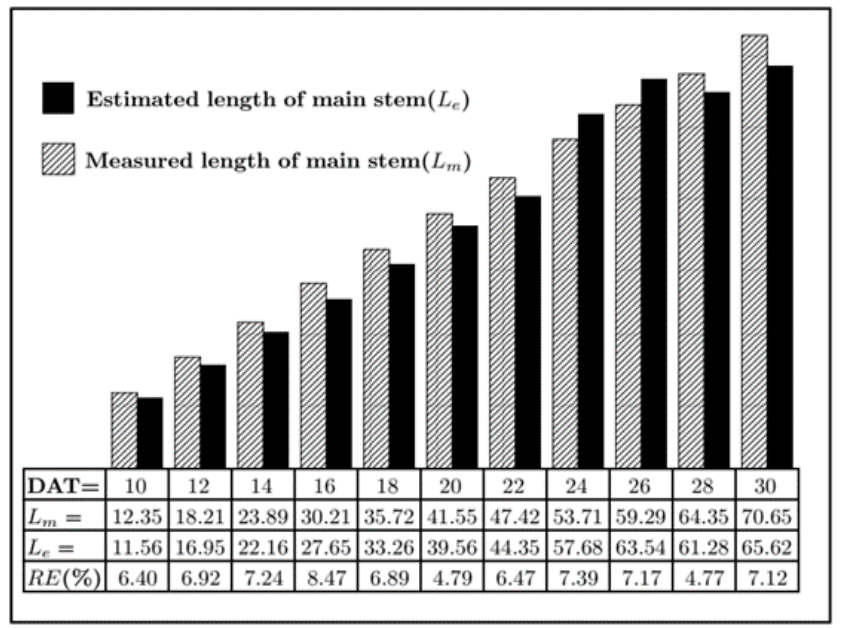

Figure 8. Estimated lengths of main stem 
Table 3. The comparision between top-view and side-view images prosessing for LA estimation

\begin{tabular}{|c|c|c|c|c|}
\hline DAT & Image view & $\mathrm{LA}_{\mathrm{e}}$ & $\mathrm{LA}_{\mathrm{m}}$ & RE (\%) \\
\hline \multirow[t]{2}{*}{10} & Top-view & 198.6 & \multirow{2}{*}{207.3} & 4.20 \\
\hline & Side-view & 199.5 & & 3.76 \\
\hline \multirow[t]{2}{*}{12} & Top-view & 242.5 & \multirow{2}{*}{255.3} & 5.01 \\
\hline & Side-view & 244.3 & & 4.31 \\
\hline \multirow[t]{2}{*}{14} & Top-view & 352.6 & \multirow{2}{*}{371.1} & 4.99 \\
\hline & Side-view & 356.2 & & 4.02 \\
\hline \multirow[t]{2}{*}{16} & Top-view & 443.0 & \multirow{2}{*}{462.9} & 4.30 \\
\hline & Side-view & 440.6 & & 4.82 \\
\hline \multirow[t]{2}{*}{18} & Top-view & 502.6 & \multirow{2}{*}{534.8} & 6.02 \\
\hline & Side-view & 498.6 & & 6.77 \\
\hline \multirow[t]{2}{*}{20} & Top-view & 577.6 & \multirow{2}{*}{613.5} & 5.85 \\
\hline & Side-view & 565.3 & & 7.86 \\
\hline \multirow[t]{2}{*}{22} & Top-view & 712.5 & \multirow{2}{*}{762.9} & 6.61 \\
\hline & Side-view & 709.6 & & 6.99 \\
\hline \multirow[t]{2}{*}{24} & Top-view & 850.3 & \multirow{2}{*}{907.5} & 6.30 \\
\hline & Side-view & 845.3 & & 6.85 \\
\hline \multirow[t]{2}{*}{26} & Top-view & 1028.3 & \multirow{2}{*}{1114.2} & 7.71 \\
\hline & Side-view & 1045.9 & & 6.13 \\
\hline \multirow[t]{2}{*}{28} & Top-view & 1210.7 & \multirow{2}{*}{1328.3} & 8.85 \\
\hline & Side-view & 1207.5 & & 9.09 \\
\hline \multirow[t]{3}{*}{30} & Top-view & 1372.9 & \multirow{3}{*}{1578.0} & 13.00 \\
\hline & Side-view & 1382.7 & & 12.38 \\
\hline & Average & & & 6.63 \\
\hline
\end{tabular}

\section{CONCLUSION}

This paper presents two new efficient approaches for cucumber leaf area estimation. In the first approach, the LA of cucumber plants in top-view images was simply estimated based on the calibration area. In this way, our proposed approach achieves a high accuracy rate while mitigating the manual camera calibration. In the second approach, three processes including background eliminating, skeleton extraction, and leaf number estimation are sequentially applied for cucumber LA estimation. Our experimental results demonstrate the effectiveness of our proposed approaches. Limitation of the paper is the fact that the quality of captured images be influenced by ambient light noise. The appearance of some overlapped leaf areas is also the main reason of high relative error. This work, however does not consider the real time estimation of leaf area. Therefore, our future plan is to enhance these issues with some realistic LA estimation algorithms.

\section{ACKNOWLEDGEMENTS}

This work has been partially funded by the project: T2019-05-04TĐ (Vietnam National University of Agriculture). The authors would like to thank the Prof. T. Sutikno for his help on organizing manuscripts, and two anonymous reviewers for their helpful and valuable comments that have greatly improved the presentation of this work.

\section{REFERENCES}

[1] M. N. I. Sarker, M. S. Islam, M. A. Ali, M. S. Islam, M. A. Salam, and S. H. Mahmud, "Promoting digital agriculture through big data for sustainable farm management," International Journal of Innovation and Applied Studies, vol. 25, no. 4, 2019.

[2] M. R. Ramli, P. T. Daely, D.-S. Kim, and J. M. Lee, "Iot-based adaptive network mechanism for reliable smart farm system," Computers and Electronics in Agriculture, vol. 170, p. 105287, 2020, doi: 10.1016/j.compag.2020.105287.

[3] M. Acosta-Coll, A. Ospino, S. Carbonell-Navarro, J. Escobar-Duque, R. Peña Gallardo, and R. Zamora-Musa, "Substrate treatment for the increment of electric power potential from plants microbial fuel cells," International Journal of Electrical and Computer Engineering (IJECE), vol. 11, no. 3, 2021, doi: 10.11591/ijece.v11i3.pp1933-1941.

[4] S. Jitanan and P. Chimlek, "Quality grading of soybean seeds using image analysis," International Journal of Electrical and Computer Engineering (IJECE), vol. 9, no. 5, pp. 3495-3503, 2019, doi: 10.11591/ijece.v9i5.pp3495-3503.

[5] L. N. Smith, W. Zhang, M. F. Hansen, I. J. Hales, and M. L. Smith, "Innovative 3d and 2d machine vision methods for analysis of plants and crops in the field," Computers in industry, vol. 97, pp. 122-131, 2018, doi: 10.1016/j.compind.2018.02.002.

[6] C. Li, R. Adhikari, Y. Yao, A. G. Miller, K. Kalbaugh, D. Li, and K. Nemali, "Measuring plant growth characteristics using smartphone based image analysis technique in controlled environment agriculture," Computers and Electronics in Agriculture, vol. 168, 2020, doi: 10.1016/j.compag.2019.105123.

[7] H. Andrianto, S. Suhardi, and A. Faizal, "Performance evaluation of low-cost IoT based chlorophyll meter," Bulletin of Electrical Engineering and Informatics (BEEI), vol. 9, no. 3, pp. 956-963, 2020, doi: 10.11591/eei.v9i3.2014.

[8] P. MR, "A Neural Network Approach to Identify Hyperspectral Image Content," International Journal of Electrical \& Computer Engineering, vol. 8, no. 4, pp. 2088-8708, doi: 10.11591/ijece.v8i4.pp2115-2125.

[9] G. Yan et al., "Review of indirect optical measurements of leaf area index: Recent advances, challenges, and perspectives," Agricultural and forest meteorology, vol. 265, pp. 2019, doi: 10.1016/j.agrformet.2018.11.033.

[10] L. K. Redden, "Plant feature detection using captured images," uS Patent, 2019, doi: 10.1016/j.agrformet.2018.11.033. 
[11] S. Azimi and T. K. Gandhi, "3d maximum likelihood estimation sample consensus for 3d correspondence grouping for plant," IEEE Sensors Letters, vol. 5, no. 6, 2021, doi: 10.1109/LSENS.2021.3075459.

[12] A. Paturkar, G. S. Gupta, and D. Bailey, "Non-destructive and costeffective 3d plant growth monitoring system in outdoor conditions," Multimedia Tools and Applications, vol. 79, no. 47, pp. 34 955-34 971, 2020, doi: 10.1007/s11042-020-08854-1.

[13] P. K. Sethy, N. K. Barpanda, A. K. Rath, and S. K. Behera, "Rice false smut detection based on faster R-CNN," Indonesian Journal of Electrical Engineering and Computer Science (IJEECS), vol. 19, no. 3, pp. 1590-1595, 2020, doi: 10.11591/ijeecs.v19.i3.pp1590-1595.

[14] A. R. Luaibi, T. M. Salman, and A. H. Miry, "Detection of citrus leaf diseases using a deep learning technique," International Journal of Electrical and Computer Engineering (IJEECS), vol. 11, no. 2, p. 1719, 2021, doi: 10.11591/ijece.v11i2.pp1719-1727.

[15] C. A. Murugan, G. Sureshkumaar, N. Kannan, and Thomas, "Bacterial foraging optimization based adaptive neuro fuzzy inference system," International Journal of Electrical and Computer Engineering (IJECE), vol. 10, no. 4, pp. 3568-3575, 2020, doi: 10.11591/ijece.v10i4.pp3568-3575.

[16] K. Yabuki, "Photosynthetic rate and dynamic environment," Springer Science \& Business Media, 2004.

[17] P. Chaudhary, S. Godara, A. N. Cheeran, and A. K. Chaudhari, "Fast and accurate method for leaf area measurement," International journal of computer applications, vol. 49, no. 9, pp. 22-25, 2012, doi: 10.5120/7655-0757.

[18] M. C. Singh, K. G. Singh, and J. P. Singh, "Indirect method for measurement of leaf area and leaf area index of soilless cucumber crop," Advances in Plants and Agriculture Research, vol. 8, no. 2, pp. 188-91, 2018, doi: 10.15406/apar.2018.08.00311.

[19] S. Bozkurt, and M. Keskin, "Effect of deficit irrigation on the estimation of cucumber leaf area under greenhouse conditions," International Journal of Agriculture \& Biology, vol. 20, pp. 877-882, 2018, doi: 10.17957/IJAB/15.0579.

[20] T. Hidayat, and R. N. Asyaroh, "Identification of plant types by leaf textures based on the backpropagation neural network," International Journal of Electrical and Computer Engineering (IJECE), vol. 8, no. 6, pp. 5389-5398, 2018, doi: 10.11591/ijece.v8i6.pp5389-5398.

[21] S. Pandey and H. Singh, "A simple, cost-effective method for leaf area estimation," Journal of botany, vol. 2011, no. 3, pp. 1-6, 2011, doi: 10.1155/2011/658240.

[22] N. J. Breda, "Ground-based measurements of leaf area index: a review of methods, instruments and current controversies," Journal of experimental botany, vol. 54, no. 392, pp. 2403-2417, 2003, doi: 10.1093/jxb/erg263.

[23] D. E. Frear, "Photoelectric apparatus for measuring leaf areas," Plant physiology, vol. 10, no. 3, pp. 569-574, 1935, doi: 10.1104/pp.10.3.569.

[24] A. R. B. Tech, A. L. C. da Silva, L. A. Meira, M. E. de Oliveira, and L. E. T. Pereira, "Methods of image acquisition and software development for leaf area measurements in pastures," Computers and Electronics in Agriculture, vol. 153, pp. 278-284, doi: 10.1016/j.compag.2018.08.025.

[25] X. Ding et al., "Changes in leaf length, width, area, and photosynthesis of fruit cucumber in a greenhouse production system," HortScience, vol. 1, no. aop, pp. 1-5, 2020, doi: 10.21273/HORTSCI14637-19.

[26] Z. Wang, K. Wang, F. Yang, S. Pan, and Y. Han, "Image segmentation of overlapping leaves based on chan-vese model and sobel operator," Information processing in agriculture, vol. 5, no. 1, pp. 1-10, 2018, doi: 10.1016/j.inpa.2017.09.005.

[27] G. Sun, Y. Li, Y. Zhang, X. Wang, M. Chen, X. Li, and T. Yan, "Nondestructive measurement method for greenhouse cucumber parameters based on machine vision," Engineering in agriculture, environment and food, vol. 9, no. 1, pp. 2016. doi: 10.1016/j.eaef.2015.06.003.

[28] D.-H. Park, H.-J. Kang, and S.-H. Kim, "A study on image processing based leaf area measurement," in Future Information Technology, Application, and Service, pp. 97-102, 2012, doi: 10.1007/978-94-007-5064-7_14.

[29] D. A. Umarhadi and P. Danoedoro, "Comparing canopy density measurement from UAV and hemispherical photography: an evaluation for medium resolution of remote sensing-based mapping," International Journal of Electrical and Computer Engineering (IJEECS), vol. 11, no. 1, pp. 356-364, 2021, doi: 10.11591/ijece.v11i1.pp356-364.

[30] N. S. Ibrahim, S. M. Sharun, M. K. Osman, S. B. Mohamed, and S. H. Y. S. Abdullah, "The application of UAV images in flood detection using image segmentation techniques," Indonesian Journal of Electrical Engineering and Computer Science (IJEECS), vol. 23, no. 2, pp. 1219-1226, 2021, doi: 11591/ijeecs.v23.i2.pp1219-1226.

[31] K. Gangadharan, G. R. N. Kumari, D. Dhanasekaran, and K. Malathi, "Detection and classification of various pest attacks and infection on plants using RBPN with GA based PSO algorithm," Indonesian Journal of Electrical Engineering and Computer Science (IJEECS), vol. 20, no. 3, pp. 1278-1288, 2020, doi: 10.11591/ijeecs.v20.i3.pp1278-1288.

[32] X. Liu, D. Zhao, W. Jia, W. Ji, C. Ruan, and Y. Sun, "Cucumber fruits detection in greenhouses based on instance segmentation," IEEE Access, vol. 7, pp. 635-139, 2019, doi: 10.1109/ACCESS.2019.2942144.

[33] G. E. Meyer and J. C. Neto, "Verification of color vegetation indices for automated crop imaging applications," Computers and electronics in agriculture, vol. 63, no. 2, pp. 282-293, 2008, doi: 10.1016/j.compag.2008.03.009.

[34] P. C. Mahalanobis, "On the generalized distance in statistics," National Institute of Science of India, 1936.

[35] C. H Chen, Image Processing for Remote Sensing, CRC Press, $2007 . \quad$ [Online]. Available: https://books.google.co.id/books?hl=id\&lr=\&id=hPQCEAAAQBAJ\&oi=fnd\&pg=PP1\&dq=Physical+Properties+of+Plant+and+ Animal+Materials:+v.+1:+Physical+Characteristics+and+Mechanical+Properties\&ots=ypowC48-

fR\&sig=BUFxVYGKQB42wNDqGrYyVIGI1Zk\&redir_esc=y\#v=onepage\&q=Physical\%20Properties\%20of\%20Plant $\% 20 \mathrm{and} \%$ 20Animal\%20Materials $\% 3 \mathrm{~A} \% 20 \mathrm{v} . \% 201 \% 3 \mathrm{~A} \% 20 \mathrm{Physical} \% 20 \mathrm{Characteristics} \% 20 \mathrm{and} \% 20 \mathrm{Mechanical} \% 20 \mathrm{Properties} \& \mathrm{f}=$ false

[36] A.-G. Manh, G. Rabatel, L. Assemat, and M.-J. Aldon, "Ae-automation and emerging technologies: Weed leaf image segmentation by deformable templates," Journal of agricultural engineering research, vol. 80, no. 2, pp. 139-146, 2001, doi: 10.1006/jaer.2001.0725.

[37] C. Xia, J. M. Lee, Y. Li, Y. H. Song, B. K. Chung, and T. S. Chon, "Plant leaf detection using modified active shape models," Biosystems engineering, vol. 116, no. 1, pp. 23-35, 2013, doi: 10.1016/j.biosystemseng.2013.06.003.

[38] A. Fabijańska and D. Sankowski, "Segmentation methods in the selected industrial computer vision application," In Computer Vision in Robotics and Industrial Applications, 2014, pp. 23-48, doi: 10.1142/9789814583725_0002.

[39] J. Khan, M. Handa, and R. Vemuri, "iPACE-V1: A Portable Adaptive Computing Engine for Real Time Applications," In International Conference on Field Programmable Logic and Applications, 2002, pp. 69-78, doi: 10.1007/3-540-46117-5_9.

[40] D. Sankowski, J. Nowakowski, D. Sankowski, and J. Nowakowski, Computer vision in robotics and industrial applications, in World Scientific Publishing Co., Inc., 2014. Online. [Avaliable]: https://books.google.co.id/books?hl=id\& $\mathrm{lr}=\& \mathrm{id}=\mathrm{kwK} 3 \mathrm{CgAAQBAJ} \& \mathrm{oi}=\mathrm{fnd} \& \mathrm{pg}=\mathrm{PR} 5 \& \mathrm{dq}=$ Computer+vision+in+robotics+and+industrial+applications\&ots=K4ze6JUDs I\&sig=x3KXpZODocLKAKh9WXjAq$\mathrm{i} 4 \mathrm{XpI} \&$ redir_esc $=\mathrm{y} \# \mathrm{v}=$ onepage $\& \mathrm{q}=$ Computer $\% 20$ vision $\% 20 \mathrm{in} \% 20$ robotics $\% 20$ and $\% 20 \mathrm{industrial} \% 20$ applications $\& \mathrm{f}=$ false

[41] G. H. Ball and D. J. Hall, "Isodata, a novel method of data analysis and pattern classification," Stanford research inst Menlo Park CA, Tech. Rep., 1965. 
[42] Z. Wang, E. Wang, and Y. Zhu, "Image segmentation evaluation: a survey of methods," Artificial Intelligence Review, vol. 53, no. 8, pp. 5637-5674, 2020, doi: 10.1007/s10462-020-09830-9.

[43] K. J. Juneau and C. Tarasoff, "Leaf area and water content changes after permanent and temporary storage," PLoS ONE, vol. 7, 2012, doi: 10.1371/journal.pone.0042604.

[44] R. Mourad, H. Jaafar, M. Anderson, and F. Gao, "Assessment of leaf area index models using harmonized landsat and sentinel-2 surface reflectance data over a semi-arid irrigated landscape," Remote Sensing, vol. 12, no. 19, 2020, doi: 10.3390/rs12193121.

[45] C. Prado and J. Moraes, "Photosynthetic capacity and specific leaf mass in twenty woody species of cerrado vegetation under field conditions," Photosynthetica, vol. 33, pp. 103-112, 1997, doi: 10.1023/A:1022183423630.

[46] B. Zheng, Y. Ma, B. Li, Y. Guo, and Q. Deng, "Assessment of the effects of leaf angle combinations on potential photosynthesis capacity of rice with 3-d models using high performance computing," in 2009 Third International Symposium on Plant Growth Modeling, Simulation, Visualization and Applications, 2009, pp. 237-244, doi: 10.1109/PMA.2009.46.

[47] N. N. Mohsenin, Physical Properties of Plant and Animal Materials: v. 1: Physical Characteristics and Mechanical Properties. Routledge, 2020. Online. [Avaliable]: https://books.google.co.id/books?hl=id\&lr=\&id=hPQCEAAAQBAJ\&oi=fnd\& $\mathrm{pg}=\mathrm{PP} 1 \& \mathrm{dq}=$ Physical+Properties+of+Plant+and+Animal+Materials:+v.+1:+Physical+Characteristics+and+Mechanical+Properti es\&ots=ypowC49XiS\&sig=h7JevZYjJeyfhKVeh0kif3EoHhg\&redir_esc=y\#v=onepage\&q=Physical\%20Properties\%20of\%20Pla nt $\% 20$ and $\% 20 \mathrm{Animal} \% 20$ Materials $\% 3 \mathrm{~A} \% 20 \mathrm{v} . \% 201 \% 3 \mathrm{~A} \% 20 \mathrm{Physical} \% 20 \mathrm{Characteristics} \% 20$ and $\% 20 \mathrm{Mechanical} \% 20 \mathrm{Properties}$ $\& \mathrm{f}=$ false.

[48] T. Zhang and C. Y. Suen, "A fast parallel algorithm for thinning digital patterns," Communications of the ACM, vol. 27, no. 3, pp. 236-239, 1984, doi: 10.1145/357994.358023.

[49] Y. Y. Cho, S. Oh, M. M. Oh, and J. E. Son, "Estimation of individual leaf area, fresh weight, and dry weight of hydroponically grown cucumbers (cucumis sativus 1.) using leaf length, width, and spad value," Scientia horticulturae, vol. 111, no. 4, pp. 330-334, 2007, doi: 10.1016/j.scienta.2006.12.028.

\section{BIOGRAPHIES OF AUTHORS}

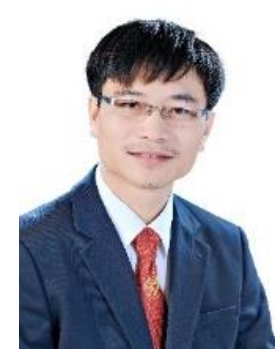

Uoc Quang Ngo (D) 8d SC P was born in 1983. He received the MA degree in electrical engineering and electrical system from the Vietnam National University of Agriculture (VNUA), Vietnam, in 2010. In 2007, he is a lecturer at the Faculty of Engineering of VNUA. $\mathrm{He}$ is currently a $\mathrm{PhD}$ student in the Institute for Control Engineering and Automation, Hanoi University of Science and Technology, Hanoi, Vietnam. His research interests include control systems, IoT, robots, and AI applications for smart agriculture. He can be contacted at: nquoc@vnua.edu.vn.

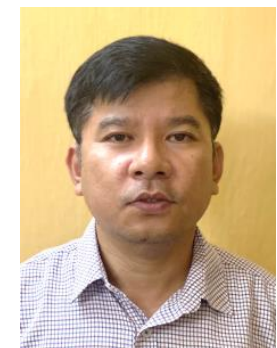

Duong Tri Ngo (iD SC S P was born in 1974. He received the PhD degree in Control Engineering and Automation from Hanoi University of Science and Technology, Vietnam, in 2009. He is currently a senior lecturer at the Faculty of Engineering of VNUA. His main research interests are control systems, IoT, and AI applications for smart agriculture. He can be contacted at: ntduongcd@ vnua.edu.vn.

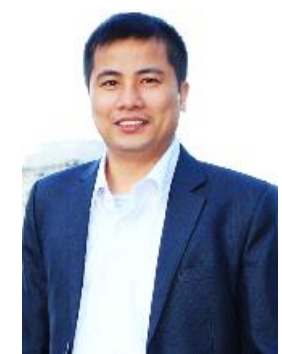

Hoc Thai Nguyen (iD) Bd SC P received his BS and MA degrees in electrical and electronic engineering from Vietnam National University of Agriculture, Vietnam in 2006 and 2010, respectively. He then received $\mathrm{PhD}$ degree in infocommunication technologies and Automation at the Budapest University of Technology and Economics (BME), Hungary in 2018. His research interests include wireless sensor networks, internet of robotic things and applications of artificial intelligence in precision agriculture. He can be contacted at: nguyenthaihoc@vnua.edu.vn and thaihocme@gmail.com.

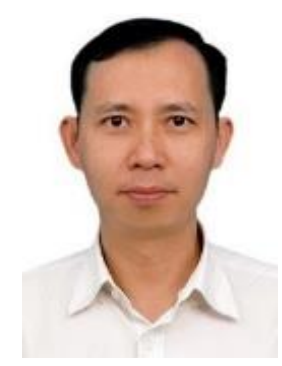

Thanh Dang Bui (D) SC P received the MA degree in Instrumentation and Control Systems from Hanoi University of Science and Technology (HUST), Vietnam, in 2001 and the PhD degree from the ENS Cachan, France, in 2011. He is currently Associate Professor at School of Electrical Engineering, HUST, Vietnam. His research interests are instrumentation system design, embedded systems, AI for smart agriculture, envirronment monitoring systems and industrial networks. He can be contacted at: Email: thanh.buidang@ hust.edu.vne. 